\title{
The importance of Midwife Runs Primary Units in Iran
} Journal of Clinical Care and Skills

\section{ART ICLE IN F O}

\section{Article Type}

Commentary Article

\section{Authors}

Alianmoghaddam N.*MSc

\section{How to cite this article}

Alianmoghaddam N. The importance of Midwife Runs Primary

Units in Iran. Journal of Clinical Care and Skills. 2020;1(1):1-3.
*School of Public Health, Massey University, Palmerston North, New Zealand

\section{Correspondence}

Address: -

Phone: +98 (64) 63569099

Fax: -

N.Alianmoghaddam@massey.ac.nz

\section{Article History}

Received: December 15, 2017

Accepted: February 11, 2018

ePublished: March 10, 2020

\section{A B S T R A C T}

This article has no abstract.

\section{I T A T I O N L INKS}

[1] Asking different questions: Research priorities to improve the quality of care for every woman, every child [2] The projected effect of scaling up midwifery [3] Babies born before arrival to hospital and maternity unit closures in Queensland and Australia [4] Prevalence and causes of cesarean section in Iran: Systematic review and metaanalysis [5] Are freestanding midwifery units a safe alternative to obstetric units for low-risk, primiparous childbirth? [6] Freestanding midwifery units: Maternal and neonatal outcomes following transfer [7] Resistance to breastfeeding: A Foucauldian analysis of breastfeeding support from health professionals [8] The closure of rural and remote maternity services: Where are the midwives? [9] The motivation and capacity to go 'above and beyond': Qualitative analysis of free-text survey responses in the M@ NGO randomised controlled trial of caseload midwifery 
The importance of Midwife Runs Primary Units in Iran

The vast majority of health research demonstrates the disadvantages of unnecessarymedical interventions in normalpregnancy and birth inlowrisk women. However, the rate of interventions is rising in the maternity systems across the world. One of the main reasons of these over-interventions in maternity system is the closure of the Midwife Runs Primary Units (MRPUs), particularly in rural andremote areas in most countries including Iran. Therefore, this commentary aims at evaluating the benefits of reopening of these MRPUs in Iran.

To improve the quality of care for each woman and her child, a recent research published in the Lancet [1] emphasises the importance of prevention of complications in maternity systems. According to Homer et al., [2] while the lack of skilled birth attendants as well as a few interventions are the significant problems in low-income countries, overintervention is contributing to morbidity and mortality in middle and high-income countries. For instance, the closure of MRPUs over the last two decades is the primary cause of increased adverse maternal and neonatal outcomes in Australia [3] and Iran [4].

In recent decades, Iran has experienced a significant increase in overall caesarean section rates. It has increased from around 20\% in 1977 to about 50\% in 2014. The caesarean section rate was even higher in private hospitals, which are reported up to $90 \%{ }^{[4]}$.

Research has shown that MRPUs could decrease the rate of caesarean sections, particularly the first-birth caesarean and promote the maternal health outcome in both primiparous and multiparous lowrisk women compared to obstetric units [5]. Monk et al. compared the birth outcomes between two groups of low-risk women, who had an intention to birth in a tertiary hospital transferred from the MRPUs [6]. The results support the optimal maternity care in MRPUs at the time of booking even for women, who were transferred to the tertiary hospital.

New Zealand has a unique midwifery system, in which women, who are at low risk of complications, are visited by a community midwife or Lead Maternity Carer (LMC) during the childbearing period. LMC midwives are paid by the New Zealand Ministry of Health and provide free maternity care for women, who are booked with them from the first trimester of pregnancy until 6 weeks postpartum. Practicing in partnership with the women is the first and most important standard of practice for each registered midwife in New Zealand [7]. Primary birth units in New Zealand are the best model of MRPUs designed for healthy pregnant women, who have no complication in their pregnancy, birth, and postpartum. MRPUs in New Zealand are staffed by Midwives only while there is an immediate access to on-call obstetricians.

Midwives are the primary care providers in maternity system and they provide cost-effective Journal of Clinical Care and Skills care, which improves the birth outcomes with minimum intervention [1]. Midwives have the adequate knowledge and skills to detect deviations from the norm and facilitate the transfer of care to secondary and tertiary healthcare settings. Therefore, they are able to practise in the primary units with basic facilities or even can manage an uncomplicated vaginal birth in a home setting [8]. Moreover, research has shown that midwife-led care can be the standard model of maternity care. It is not only the best option for the low-risk pregnant women, but the safe and cost-effective model of care for all women of any risk. The researchers also pointed out that the benefits of midwife-led care are not limited to only women and their families, and its outcome is beneficial for the health service as well as the national economy [9].

However, the midwifery profession has recently been described as in crisis, with midwifery care characterized as undervalued and under-resourced, evidenced through high staff turnover, and low midwifery workforce retention rates [7]. In addition, in Iran, still ismassive opposition to the midwiferyled model of care to some extent that most state hospitals' policies do not support collaborative arrangements with self-employed midwives.

In conclusion, this paper asks the question, "Is it necessary to reopen the midwife runs primary units in Iran?

\section{References}

1- Kennedy HP, Yoshida S, Costello A, Declercq E, Dias MA, Duff E, et al. Asking different questions: Research priorities to improve the quality of care for every woman, every child. Lancet Glob Health. 2016;4(11):e777-9.

2- Homer CS, Friberg IK, Dias MA, ten Hoope-Bender $\mathrm{P}$, Sandall J, Speciale AM, et al. The projected effect of scaling up midwifery. Lancet. 2014;384(9948):1146-57.

3- Kildea S, McGhie AC, Gao Y, Rumbold A, Rolfe M. Babies born before arrival to hospital and maternity unit closures in Queensland and Australia. Women Birth J Aust Coll Midwife. 2015;28(3):236-45.

4- Azami-Aghdash S, Ghojazadeh M, Dehdilani N, Mohammadi M, Asl Amin Abad R. Prevalence and causes of cesarean section in Iran: Systematic review and meta-analysis. Iran J Public Health. 2014;43(5):545-55.

5- Christensen LF, Overgaard C. Are freestanding midwifery units a safe alternative to obstetric units for low-risk, primiparous childbirth?. An analysis of effect differences by parity in a matched cohort study. BMC Pregnancy Childbirth. 2017;17(1):14.

6- Monk AR, Grigg CP, Foureur M, Tracy M, Tracy SK. Freestanding midwifery units: Maternal and neonatal outcomes following transfer. Midwifery. 2017;46:24-8.

7- Alianmoghaddam N, Phibbs S, Benn C. Resistance to breastfeeding: A Foucauldian analysis of Winter 2020, Volume 1, Issue 1 
breastfeeding support from health professionals. Women Birth. 2017;30(6):281-91.

8- Barclay L, Kornelsen J. The closure of rural and remote maternity services: Where are the midwives?. Midwifery. 2016;38:9-11.
9- Allen J, Kildea S, Hartz DL, Tracy M, Tracy S. The motivation and capacity to go 'above and beyond': Qualitative analysis of free-text survey responses in the M@ NGO randomised controlled trial of caseload midwifery. Midwifery. 2017;50:148-56. 